2. As soon as the cold weather commences both, to a grest extent, become extinct. Daring the late summer and fall months of 1909, while we had a severe epidemic of typhoid fever in Montreal amongst the adults and young people, at the same time we had an epidemic of poliomyelitis amongst the young children and babies.

3. Diarrhoea is just as common a symptom in poliomyelitis as in typhoid fever.

4. The incubation period of one is practically the same as that of the other. This has been proved by Dr. Flexner to be thirteen days experimentally, and Dr. Holt found it to be ten days clinically.

5. Both diseases are prevalent in large cities and small towns at the same time where and when the cause of typhoid fever is traceable to the water and milk supply whilst, on the other hand, where the supply of water and milk is pure, both of these diseases are less frequent. It is practically the same degree of contagion.

These observations led me to believe that the microorganism of poliomyelitis might be of the same character as the bacillus of typhoid fever.

Upon examination of the blood in cases suffering from poliomyelitis, I have found the Widal reaction present as in typhoid fever, proving conclusively that the disease is from the same source and cause, and that the germ of the one is allied to that of the other; further, I do not hesitate to make the assertion that the precautions necessary to prevent typhoid fever will also have the same effect in arresting the spread of poliomyelitis.

It will be seen that I obtained the Widal reaction in 6 cases of the blood examination out of a total of 16 cases. In four instances the report is highly suggestive, while in 6 cases the result was negative. However, three of these were taken from individuals in good health to prove that the Widal reaction was not a family pecaliarity. This only leaves 3 negative cases to deal with. Two of the negative results were obtained from cases of poliomyelitis about two and a half and three months from the time of onset of the disease, and both of the cases were mild in character, only one leg being partially piralysed.

It appears to me that these results correspond fairly well with the results which would be obtainable from the uxamination of a similar number of typhoid patients. I may add that the blood was examined in every case by physicians who make a speciality of this wark.

As typhoid fever affects the most vulnerable part of the adult-the intestinal tract, so poliomyelitis attacks the weakest point of resistance in the child, which is the nervous system.

As to treatment, there is no doubt that within the near future a serum will be obtained; and, if so, in suspected cases, during an epidemic of poliomyelitis, it is well to look for the Widal reaction, and, if present, the serum should be injected, which should have the effect of either modifying or preventing the development of the disease. In cases in which the disease has already developed, to prevent further extension the serum might be injected into the spinal canal, and would be likely to have an effect aimilar to that obtained by the method of treatment in corebro-spinal meningitis.

REFERENCES.

1 Editorial note, Archives of Pediatrics, February, 1910, 2 New York Academy of Medicine, Section on Poliomyelitis, January 20th, 191C.

THE late M. Auguste Loutreuil, a Frenchman who made a large fortune by industrial enterprise in Russia, has bequeathed $£ 120,000$ to the Académie des Sciences, $£ 100,000$ to the Sorbonne, $£ 40,000$ to the Fund for Scientiflc Research, and $£ 4,000$ to the Paris Parteur Institute. The money is intended to be applied in furtherance of scientific research.

IT was recently decided to form a Plymouth. West Devon, and Cornwall branch of the Research Defence Society, a Devon branch already existing under the presidency of Mr. Eden Phillpotts. The following are offeers of the branch: President, Mr. W. Paul Swain; vice.presidents, Admiral Marrack, Colonel Giles, and the Rev. Dr. Trelawney Ross; honorary secretary, Dr. R. Jaques; honorary treasurer, Mr. Cyril A. Pode; committee, the president, honorary secretary, honorary treasurer, Miss Kirk, Colonel Giles, with two others jet to be appointed. A ladies' committee is to be formed.

\section{THE GASTRIC ORIGIN OF ANGINA PECTORIS.}

BY

\section{H. WALTER VERDON, F.R.C.S.ENG.}

ON January 2nd, 1911, when I entered Dr. X.'s room he was suffering from an anginal seizure which had already lasted half an hour. He had been making ineffectaal efforts to expel wind from his chest, and allowed me to introduce an oesophageal tabe, a procedure which resulted in an exhanstive liberation of gastric flatus. The effects of the operation became instantly apparent in the altered aspect of the patient. His colour returned, and he seemed no longer in pain. The seizure was manifestly at an end. On the last day of January Dr. X. informed me that on two occasions he had again resorted to the operation of gastric deflation, and upon each he had brought the seizure to a sudden and abrupt close. Dr. $X$. is 63 years of age, and has suffered from anginal seizures from time to time for several years. His first severe attack occurred in 1908.

When a seizure bas been brought to an abrupt and com. plete termination by the introduction of the oesophageal

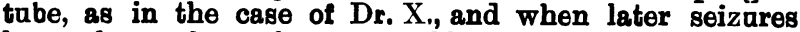
have shown themselves amenable to a similar operation, the fact that the seizures are the resalt of tension of the gastric atmosphere is demonstrated beyond question.

Admission of this fact involves the recognition of the theory of the gastric reflex in the case of Dr. $X_{\text {., and also }}$ having regard to all cases of the type to which Dr. X.'s seizures genetically belong.

This type appears to be a prevalent one, since in descriptions of anginal seizures gastric symptoms are often mentioned as holding a prominent place. Time after time in an account of this disease Dr. James Mackenzie refers to flatulent distension, and even propounds the theory of air.swallowing to account for it. Moreover, Heberden in his classic description states that the seizure comes on more particularly when the patient walks after $\epsilon$ ating.

Probably in the same category are to be classed cases dying in an anginal seizure when at the autopsy no traces of disease in the coronary arteries are found. These number nearly 50 per cent. in a long series of collected cases.

If this be so, the theory of the gastric reflex must be admitted to occupy at least an equal place in our regard with that of the cardiac reflex.

Recently a case came under my notice demonstrating the fact that under certain favouring circumstances tension of the gastric atmosphere is associated with painful attacks indistinguishable from those of angina pectoris.

Mr. M., aged 62, for many years a sabject of flatulent dyspepsia, had lately lost flesh, and in the course of his wasting had developed seizures of a painful character. The pain affected him when he walked and on going apstairs. It was felt in the region of distribution of the upper intercostal nerves including the costo-humeral branch. The seizures came on earlier in the walk after he had taken food than when he fasted, they were associated with sensations of gastric distension and they subsided upon free eructation of flatus. Seizures of a similar character often awakened him from sleep, and alike were relieved by expulion of gas from the stomach. Mr. M. had relieved by expulion of gas from the stomach. Mr. M. had walked and disturbing his rest at night, bat never before had they been accompanied by pain. Since pain had grown severe morphine had been taken, but with no satisfactory result. In this case the exhibition of nitroglycerine* proved to be exceedingly effective in the relief of pain, seizures always subsiding immediately after its administration, and its efficiency remained unimpaired until the day of his death, which occurred remained unimpaired until the day of his death, which occurred revealed the presence of carcinoma which had infiltrated the wall of the oesophagus in the lower third of its length. There were secondary growths in the coeliac glands and in the neighbourhood of the liver. The oesophagus was slightly dilated in the region corresponding with carcinomatous infiltration and the cardiao oriflce was patent. The heart, coronary arteries, and systemic vessels were quite healthy.

The seizures of pain in this case might be explained as being the result of pressure from a tense gastro-0t80pha. geal atmosphere transmitted through the oesophageal wall and in pioging upon the fibres of the plexus galse. The plexus galae surrounds the oesophagus in tbe lower

* The action of the nitrites is upon every kind of plain muscle throughout the body (Dixon's Pharmacology, p. 197). 
part of its course, and its deep border lies an inch or more above the fusion of this tube into the stomach. It is formed by the breaking up of the vagi trunks into their constituent fibres, which anastomose at points and build up a network or cage which envelops the gullet; at the lower margin of the plexus the fibres converge and are again formed into two separate bundles. This plexus is reinforced by filaments from the sympathetic chain, and some of these filaments are of peculiar interest.

Sympathetic filaments worthy of special note arise in the second, third, and fourth thoracic segments of the spinal cord. They leave the spinal cord in connexion with the intercostal roots derived respectively from these seg. ments and pass into the sympathetic chain; thence they proceed to the posterior pulmonary plexuses, ${ }^{1}$ and when in this situation join the diverging fibres of the vagi as they descend to form the plexus gulae.

The significance of this sympathetic connexion lies in the fact that incidence of pain in the seizures from which Mr. M. suffered occurred in the cutaneous distributing area of the second, third, and fourth intercostal nerves, whose roots are associated with these flaments having connexions with the plexus gulae.

The paroxysmal nature of the seizure is also explain. able on this hypothesis. Stimulation of the vagi results in contraction of the oesophagas, contraction of the pylorus, opening of the cardiac orifice of the stomach, and contraction of gastro-mural muscle ${ }^{2}$-in other words, imprisonment of the gastro-0esophageal atmosphere under pressure. A sequence of gastro-circulatory symptoms is thus set up, which only ends upon the escape of flatus or relaxation of gastro-mural muscle.

The case of Mr. M. therefore demonstrates the manner in which painful seizures of an anginal character may occur as a result of gastric tension.

Arguments in favour of the theory of a cardiac reflex are chiefly based on the doctrine of intermittent claudication introduced into the literature of angina pectoris by Benjamin Brodie in the year 1846. It is asserted that funotional or organic obstruction of the coronary arteries, incomplete or complete, by narrowing the blood stream flowing towards the myocardium, or by withholding it altogether, is the cause of the seizure. Upon first impressions the doctrine looks probable enough; moreover, it is persuasive, since it can be made to apply in all cases. When death occurs during a seizare impacted emboli may be found in the coronary arteries, or these vessels may be thrombosed by blood clot of old or recent formation. Then the cause of death is obvious, and the seizure is accounted for. If these morbid conditions be absent and a state of arterio-sclerosis alone exists, it is argued that the arterial supply is sufficient while the heart is acting under normal conditions, but, when its action is increased by walking exercise or mental excitement, the supply falls short of the angmented needs of the myocardium, and consequently a seizure occurs. Nor is there any difficulty in accounting by this hypothesis for the 49.9 per cent. of cases belonging to a series introduced below, in which the heart and coronary arteries are found at the autopsy in a healthy state without sign, either macroscopic or microscopic, of any trace of organic disease, for even in regard to these cases, which might . be considered by some as constituting a weak point in the orthodox doctrine of cardiac reflex by claudication, the student is reminded that functional spasm may exist in the coronary vessels as in any other in the circulatory economy. Since the days of Sir Benjamin Brodie these arguments have approved themselves to writers of textbojks and teachers of schools, and I do not remember noticing anywhere that they have been under question. Yet I do not think they can survive even a superficial examination, since a result of recent investigations into the physiology of the coronary arteries pub. lished by Schäfer ${ }^{8}$ shows that the coronary arteries are not endowed with any vasomotor mechanism, nor do they suffer vaso-constriction in the presence of any known stimulus. It is idle, therefore, to continue to speak of functional spasm of these vessels as a cause of the seizure. Thus, by a stroke of the pen, 49.9 per cent. of anginal cases on the list of autopsies introduced below have already ceased to repose under the sanction of the doctrine of clandication. What of the 50.1 per cent. of anginal cases in which coronary disease is found at the autopsy? Do statistics warrant the assertion that coronary disease is a pre- disposing canse of angina pectoris? This question can be answered by a consideration of two further questionsnamely, Is coronary disease more frequently found in autopsies made on anginal subjects than in autopsies made on other classes of circulatory disorder? And another, Is the age-period of life in which coronary disease is most frequently found also the age-period at which angina pectoris claims the greatest number of victims?

Taking under consideration a long series of autopsies in angina pectoris, numbering 283 cases collected by Tacchi, Sir John Forbes, Lusani, Potain, Gothair, Huchard, and Osler, I find a percentage of 50.1 in which coronary disease was present, and a percentage of 49.9 in which coronary disease was absent.

Compare this series with a list of 398 cases of arterial disease affecting indifferent visceral arteries, collected by Dr. Harlow Brooks, assistant professor of pathology, Bellevae Hospital and University, New York. These cases are described as coming under his notice consecutively in the ordinary course of his necropsial daties, the average age-period being 45 years. Brooks observed in this list 270 instances of coronary disease. This number represents a ratio equal to 73.3 per cent. of coronary disease. ${ }^{4}$ It is thus evident that the prevalence of coronary disease in angina pectoris does not exceed that found in another class of circulatory disease. Brooks also states that of all visceral arteries the coronaries are far more frequently affected than any other, and that in aged persons coronary disease is almost universal.

Moreover, if coronary disease be a predisposing or excit. ing cause of angina pectoris, the death incidence of angina. pectoris might be expected to increase annually after 60 . pears of age, when year by jear the incidence of coronary disesse is bounding upwards. But, just when the incidence of angina pectoris ought to be rising, in accord. ance with the accepted hypothesis, it is falling.

Osler gives the age-periods of mortality from angina pectoris. I quote from his Lumleian Lectures on the subject :

There were under 30 years of age, 9 cases; between 30 and 40 , 41 cases; between 40 and 50,59 cases; between 50 and 60,81 cases; between 60 and 70, 62 cases ; between 70 and 80, 13 crses ; above 80,3 cases. ${ }^{5}$

According to Osler's figures, which correspond fairly accurately with those of the Registrar.General; the inci. dence of death from angina pectoris after 60 years of age declines year by year, until at 80 it approaches nullity, whereas during the same age-periods the incidence of coronary disease is increasing year by year until it reaches universality.

It is thus gathered from statistical evidence that the effect of the incidence of coronary diserse on the causation of true angina pectoris of Heberden is nil. Nevertheless, its influence in the determination of a fatal issue is enor. mous; a myocardium impoverished through the attenuation of its nutrient supply soon falls victim to the exigen. cies of an anginal seizure. Nor is it to be wondered at if during this period of cardiac disturbance diseased coronary vessels become obstructed with thrombus or embolus.

In accordance with this argument, thrombus and embolus of the coronary arteries, and even ruptare of the ventricle, are the result and not the cause of the anginal seizure.

Moreover, when these accidental or cataclysmal pheno. mena spontaneously occur, the seizare resulting therefrom, although painfal and fatal, does not belong to the anginal category; and finally, all cases presenting the true paroxysmal characteristics as described by Heberden are probably the result of a gastric reflex, and perchance may be made amenable to gastric treatment by surgical methods, palliative or radical.

1 Cunningham's Anatomy, REFERENCEs.

等 Med. Sci., May, 1906, p. 781. E Lancet, March 12th, 1910, p. 698.

UNDER the will of the late Mr. Henry Veasey of Aspley Guise, Bedfordshire, the Royal Medical Benevolent Fund and the Hunstanton Convalescent Home receive $£ 300$ and $£ 200$ respectively. The testator, who died in January at the age of 92, was a medical man formerly in practice in the village in which he died. He received his medical education at Guy's Hospital, becoming L.S.A., and M.R.C.S. in the year 1840 and F.R.C.S. in the year 1862. His estate was valued at $£ 36,479$. 